\title{
Anisotropic charge displacement supporting isolated photorefractive optical needles
}

\author{
Eugenio DelRe \\ Advanced Research, Pirelli Cavi e Sistemi, Viale Sarca 222, 20126 Milan, Italy \\ Alessandro Ciattoni \\ Dipartimento di Fisica, Università dell'Aquila, and INFM, Unità di Roma 1, Rome, Italy \\ Aharon J. Agranat \\ Department of Applied Physics, Hebrew University of Jerusalem, Jerusalem 91904, Israel
}

(October 25, 2018)

\begin{abstract}
The strong asymmetry in charge distribution supporting a single non-interacting spatial needle soliton in a paraelectric photorefractive is directly observed by means of electroholographic readout. Whereas in trapping conditions a quasi-circular wave is supported, the underlying double-dipolar structure can be made to support two distinct propagation modes.
\end{abstract}

Far from being a peculiarity of low dimensional systems, solitary waves and solitons have been widely documented in bulk three-dimensional environments [1]. In biased photorefractives, nonlinear visible optical waves have been shown to undergo self-trapping both as extended one-dimensional waves, in the form of slabsolitons [2], and as confined two-dimensional spatial beams, needle-solitons [3]. These are self-funneled micron-sized beams of light that propagate through the bulk dielectric without suffering diffraction or distortion. Needles, in their richer higher-dimensional environment, have led to a substantial advance in our phenomenological investigation of nonlinear dynamics, expanding the scope of possible soliton-based applications [- $[$ [ [5].

Whereas both slabs and needles emerge in the same physical system, a biased photorefractive sample, their underlying nonlinear nature is rather different [6]. For slabs, the entire physical system, and thus, consequently, the optical nonlinearity, depends only on the transverse beam direction along which the external field is applied (say the $\mathrm{x}$ direction), whereas the system is fully invariant for spatial translations in the second orthogonal transverse direction y. This reduces slab soliton description to that associated with a saturated Kerr-like nonlinearity [7]. For needles, on the contrary, the higher dimensionality of the optical beam, whose quasi circular symmetry suggests an isotropic self-action [3], is inherently at odds with the screening nonlinearity, whose one basic driving mechanism is the $\mathrm{x}$ directed external bias field. A simplified description of needles, tracing the steps that lead to a local Kerr-like understanding of slabs, is simply not possible [8]. Given the complexity of the higherdimensional interaction, the theoretical interpretation of needles is largely based on numerical integration. What emerges is a picture in which nonlocal nonlinear effects [9], as opposed to local conventional paradigm Kerr-like phenomenology, play a central role. An understanding of these requires an explicit distinction between the underlying space-charge field distribution $E_{s c}$, which mediates self-action, and the propagating light field $E_{\text {opt }}$. The space-charge distribution simply does not have a local relationship to the optical field [8]. The numerical solution of the full boundary-value problem indicates that the highly anisotropic screening configuration allows the formation of needles only through an equally anisotropic local space-charge, characterized by the appearance of two distinct lateral field lobes in the $\mathrm{x}$ direction, absent in the second transverse direction y [6] [9]. This double dipolar field distribution induces, as a consequence, a complicated needle supporting index pattern that has little to do with a mere self-written graded-index waveguide (excluding the possibility of a simple linear interpretation [10]). For system parameters far from the soliton supporting configuration, this anisotropy leads to an observable asymmetric beam distortion, but the question naturally arises as to how these lobes manifest their existence when the optical beam is actually a needle-like solitary wave.

Repulsion of mutually incoherent needles provides indirect evidence of the lobe-like charge distribution [11]. However, no direct experimental evidence of charge anisotropy has yet been reported. The main reason lies in the fact that photorefractive solitons are generally observed in ferroelectric samples. In these crystals there is no direct way of isolating the contribution of charge displacement from the final guiding structure. Readout with non-photorefractively active light can lead to no substantial increase in knowledge on the underlying charge pattern, short of performing precise bulk interferograms or far field soliton transforms [9]. Direct investigation of the space-charge residue with a probe is furthermore hampered by the fact that the lobes are actually antiguiding [6] [9].

In this Letter we give direct evidence of this nonlocal 
field structure. This is made possible by the quadratic electro-optic response of paraelectrics, that allows the electro-holographic separation of optical phenomenology from the underlying space-charge field [5].

Experiments are carried out in a sample of photorefractive $3.7^{x} \times 4.7^{y} \times 2.4^{z} \mathrm{~mm}$ KLTN (potassium-lithiumtantalate-niobate) [12], biased along the $\mathrm{x}$ axis (of size $\mathrm{L}=3.7 \mathrm{~mm})$, and kept at a constant temperature $\mathrm{T}=20^{\circ} \mathrm{C}$. The x-polarized cw TEM $\mathrm{TE}_{00} \lambda=532 \mathrm{~nm}$ beam from a diodepumped doubled NdYag laser is focused on the input facet of the sample and launched along the $\mathrm{z}$ axis. As it propagates in the sample, it diffracts, passing from an initial intensity $\mathrm{I}=\left|E_{\text {opt }}\right|^{2}$ full-width-half-maximum (FWHM) in the $\mathrm{x}$ and $\mathrm{y}$ directions $\Delta x \cong \Delta y \cong 10 \mu \mathrm{m}$ to a broadened intensity distribution of $\Delta x \cong \Delta y \cong 20 \mu \mathrm{m}$ (see Fig1.(a),(b)). The application of the external constant bias $\mathrm{V}$ on the $\mathrm{x}$ electrodes makes photoexcited free charges drift, leading to an inhomogeneous field screening. The electro-optic response of the paraelectric sample is $\Delta n=-(1 / 2) n^{3} g_{11} \epsilon_{0}^{2}\left(\epsilon_{r}-1\right)^{2}(V / L)^{2}(E /(V / L))^{2} \equiv$ $-\Delta n_{0} \mathcal{E}^{2}$, where $n \cong 2.4$ is the zero-field index of refraction, $g_{11} \equiv g_{x x x x} \cong 0.12 \mathrm{~m}^{4} C^{-2}$ is the dominant component of the quadratic electro-optic tensor $\mathrm{g}_{i j k l}$ (and thus tensorial effects are neglected), $\epsilon_{0}$ is the vacuum dielectric constant, $\epsilon_{r} \cong 9 \cdot 10^{3}$ (at $\mathrm{T} \cong 20 \circ \mathrm{C}$ ) is the relative sample low frequency dielectric constant, $E$ is the $\mathrm{x}$ component of the electric field resulting from screening, $\Delta n_{0} \cong 2.8 \cdot 10^{-4}$, and $\mathcal{E} \equiv E /(V / L)$. The spatially modulated index distribution allows needle formation (see Fig.1(c)). The needle, that shows a slight anisotropy in the output intensity distribution, is trapped and stable in time for an external bias voltage of $\mathrm{V}=0.85 \mathrm{kV}$ and a ratio of peak intensity $\mathrm{I}_{p}$ to the dark artificial illumination $\mathrm{I}_{b}$ (obtained by illuminating the sample with a copropagating y polarized plane wave of equal wavelength) of $\mathrm{u}_{0}^{2} \equiv I_{p} / I_{b} \cong 2.6$. Annulling the externally applied voltage $\mathrm{V}$, i.e., setting $\mathrm{V}=0$, gives an index modulation $\Delta n_{V=0}=-\Delta n_{0} \mathcal{E}_{s c}^{2}$, only due to the charge displacement, where evidently $\mathcal{E}{ }_{s c} \equiv \mathcal{E}$-1. The resulting index pattern has a guiding structure for regions in which $\mathcal{E} s c$ passes through a minimum. Given that the lobes represent an excess of screening in the $\mathrm{x}$ direction [6] [9], there are two points, i.e., $\mathrm{x}_{1}$ and $\mathrm{x}_{2}$, along the $\mathrm{x}$ axis, located to the left and right of the needle peak, in which $\Delta n_{V=0}$ forms a guiding "hump". Along the y axis, this hump will follow the shape of the lobe.

In order to investigate $\Delta n_{V=0}$ without modifying the space-charge distribution, we launch into the sample the same beam leading to the needle, but attenuated so as to have a much lower intensity. This guarantees that the characteristic time scale of charge displacement induced by the probe, $\tau_{d}$, is much longer than any characteristic observation time. For typical $\mu \mathrm{W}$ intensity beams, $\tau_{d} \sim 1$ $\min$.

Results, shown in Fig.1(d), clearly indicate the anisotropic lobe structure in the form of a split diffrac- tion pattern in the $\mathrm{x}$ direction. The slight asymmetry in the diffraction pattern is a consequence of needle selfbending, that inevitably distorts the diffractive read-out phase.
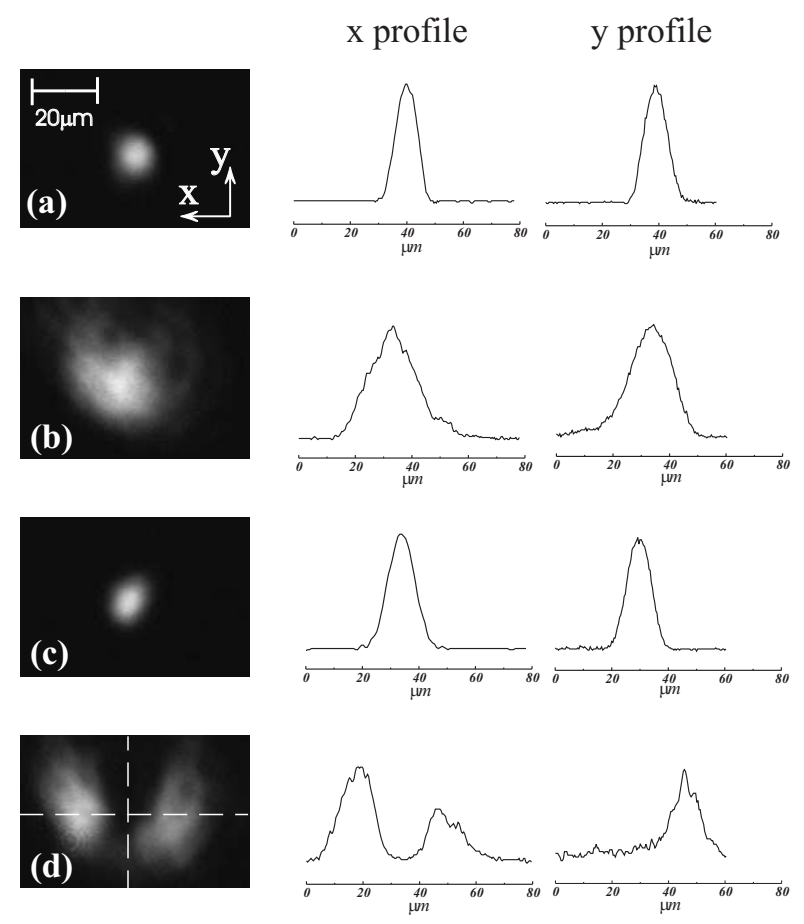

FIG. 1. Electroholography of a single photorefractive needle. (a) Image and profiles of input transverse intensity distribution; (b) Linear diffraction with nonlinear charge separation turned off $(\mathrm{V}=0)$; (c) Self-trapping distribution for $\mathrm{V}=0.85 \mathrm{kV}$; (d) Read-out for $\mathrm{V}=0$.

A similar phenomenology has been observed for transient quasi-steady-state needles, where $\mathrm{I}_{b}=0$, blocking beam evolution in the trapped regime, i.e., before the needle has decayed.

The two light lobes are a signature of the lobes predicted by numerical integration of the full Kukhtarev model and constitute direct proof that the nonlinearity supporting needle trapping in biased photorefractives is not the saturated Kerr-like $\Delta n \propto 1 /\left(1+I / I_{b}\right)^{2}$ that allows slab formation. More precisely, whereas the lobes are not present in the slab case (and are not merely "negligible"), they play a fundamental role in needle trapping [13]. Although needles have been documented in various conditions, it is legitimate to ask whether the nonlocal space-charge field structure, and thus index modulation, can actually support circularsymmetric solitary waves. The mathematical answer is no [14]. However, the anisotropic space-charge structure can support waves that are to all practical purposes circular-symmetric. For the conditions investigated ex- 
perimentally, we find the space-charge distribution by solving the simplified associated electro-static problem, i.e., $\nabla \cdot\left[\left(I+I_{b}\right) \mathbf{E}+\left(K_{b} T / q\right) \nabla I\right]=0$, where $\mathbf{E}[8]$ is the internal electric field vector, assuming a given Gaussian intensity distribution. The resulting index pattern is shown in Fig.(2a). Propagating the very same field distribution $E_{\text {opt }}$ (whose intensity is I, shown in Fig.(2b)) into this pattern, gives results shown in Fig.(2c)-(2d). The intensity pattern does not suffer discernible distortion. This means that the exact nonlinear behaviour is well described by this approximate linear approach, and thus we can conclude that quasi-circular needles can be supported by the anisotropic pattern.
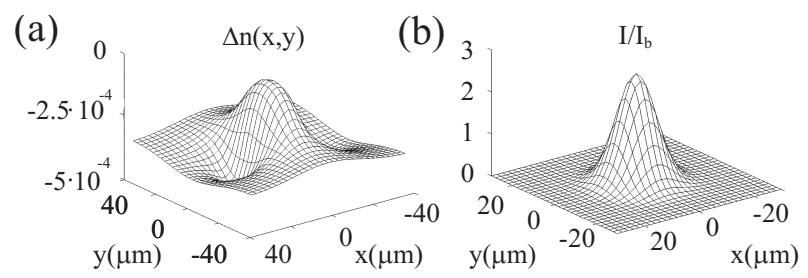

(c)
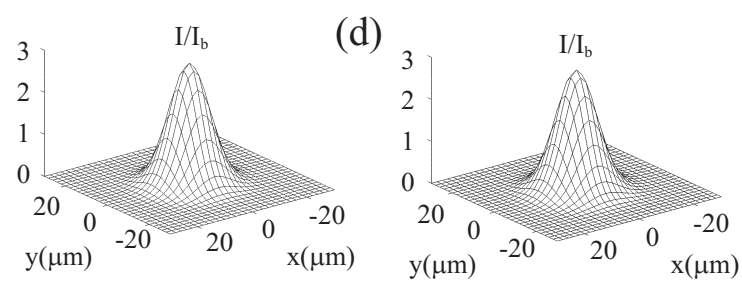

FIG. 2. Self-consistency of a needle solitons trapped in the anisotropic nonlinear index pattern. (a) Anisotropic index pattern; (b) input intensity distribution; (c)-(d) Intensity after $2 \mathrm{~mm}$ and $4.5 \mathrm{~mm}$ propagation, respectively, for the experimental situation described above.

One basic consequence of these findings is that the anisotropy underlying a photorefractive needle leads not to one, but to three spatially separated index structures, that can be made to alternatively guide light depending on the applied external voltage in the read-out phase. This would not have been possible had the nonlinear response been local, as in the one-dimensional case [5]. The electroholographic read-out would have implied a transition from a localized single mode structure (the needle) to a delocalized "doughnut-like" guiding pattern. To demonstrate this, we investigate the guiding capabilities at $\mathrm{V}=0$. We were able to show the two guided modes launching, in sequence, the probe beam into one of the two lateral guiding humps of the $\Delta n_{V=0}$ pattern, i.e., in $x_{1}$ and $x_{2}$. Results are shown in Fig.3. We did not observe any directional coupling between the modes, this clearly being a consequence both of the distance between the humps, the probe wavelength, propagation length, and the presence of the antiguiding central pattern.

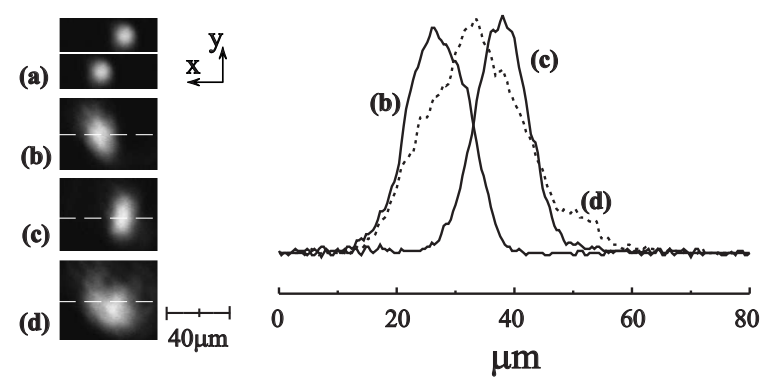

FIG. 3. Double hump guiding structure. (a) Two different input beams, shifted by approximately $\pm 10 \mu \mathrm{m}$; (b-c) Guided beam in the two humps; (d) Linear diffraction for unshifted beam.

The work of E.D. was partially carried out during previous activity at Fondazione Ugo Bordoni. The work of A.C. was funded by the Istituto Nazionale Fisica della Materia through the PAIS2000 SESBOM project. Research carried out by A.J.A. is supported by a grant from the Ministry of Science of the State of Israel.

[1] G.I. Stegeman and M. Segev, Science 286, 1518 (1999); M. Segev and M. Stegeman, Phys. Today 51, 42 (1998).

[2] M. Segev, B. Crosignani, A. Yariv, B. Fischer, Phys.Rev.Lett. 68, 923 (1992).

[3] M. Shih, M. Segev, G.C. Valley, G. Salamo, B. Crosignani, P. Di Porto, Electron. Lett. 31, 826 (1995).

[4] S. Lan, M. Shih, G. Mizell, J. A. Giordmaine, Z. Chen, C. Anastassiou, J. Martin, and M. Segev, Opt. Lett. 24, 1145 (1999); S. Lan, E. DelRe, Z.G. Chen, M.F. Shih, M. Segev, Opt. Lett. 24, 475 (1999).

[5] E. DelRe, M. Tamburrini, A.J. Agranat, Opt. Lett. 25, 963 (2000).

[6] A.A. Zozulya and D.Z. Anderson, Phys.Rev. A 51, 1520 (1995).

[7] M. Segev, M. Shih, G.C. Valley, J.Opt.Soc.Am.B 13, 706 (1996).

[8] B. Crosignani, P. Di Porto, A. Degasperis, M. Segev, and S. Trillo, J.Opt.Soc.Am.B 14, 3078 (1997).

[9] C.M. Gomez Sarabia, P.A. Marquez Aguilar, J.J. Sanchez Mondragon, S. Stepanov, and V. Vysloukh, J.Opt.Soc.Am.B 13, 2767 (1996).

[10] A.W. Snyder, D.J. Mitchell, L. Poladian, and F. Ladouceur, Opt.Lett. 16, 21 (1991).

[11] W. Krolikowski, M. Saffman, B. Luther-Davies, and C. Denz, Phys.Rev.Lett. 80, 3240 (1998).

[12] A.J. Agranat, R. Hofmeister, and A. Yariv, Opt. Lett. 17, 713 (1992).

[13] There is no fundamental difference between anisotropy in paraelectrics and ferroelectrics.

[14] M. Saffman and A.A. Zozulya, Opt.Lett. 23, 1579 (1998). 